\title{
La protéine amyloïde A, son précurseur sérique et l'amylose A
}

\section{Les nouvelles de ce numéro ont été préparées par: Jean-Claude Dreyfus Jean-Pierre Grünfeld Axel Kahn Marc Peschanski}

L'amylose A complique l'évolution de certaines suppurations prolongées, de certaines maladies inflammatoires chroniques et de maladies génétiques comme la fièvre méditerranéenne familiale (ou maladie périodique). La protéine amyloïde A (AA) constitue les fibrilles caractéristiques de la substance amyloïde qui se dépose dans les tissus; on connaît le précurseur de cette protéine, la protéine sérique AA (ou SAA).

En fait, plusieurs protéines AA ont été caractérisées et la plupart d'entre elles correspondent aux 76 premiers acides aminés de la SAA. La partie carboxyterminale de la molécule SAA est polymorphe : deux formes principales et quatre formes mineures ont été identifiées. En revanche, la portion moyenne de la molécule de protéine AA est conservée et fixe dans les espèces étudiées [1].

Dans le sérum d'un malade, Kluve-Beckerman et al. [2] ont trouvé trois SAA différentes, deux formes $\left(\mathrm{SAA}_{2} \alpha\right.$ et $\left.\mathrm{SAA}_{2} \beta\right)$ distinctes par un seul acide aminé en position 71 , et une autre $\left(S A A_{1}\right)$ avec plusieurs substitutions. Ils ont identifié les trois $\mathrm{ARNm}$ codant pour ces protéines; le polymorphisme observé en position 71 est également présent dans l'ADN ; il n'est donc pas dû à un événement survenu au cours de la traduction. Chez l'homme, les gènes de la SAA n'ont pas été complètement caractérisés, mais au moins deux gènes sont présents, codant pour des SAA, et ont été localisés au bras court du chromosone $11[1,2]$.

Les maladies inflammatoires et les infections bactériennes récidivantes (comme au cours de la mucoviscidose) s'accompagnent d'une production accrue de SAA. La transcription des gènes de SAA est déclenchée par l'interleukine-1 (IL-1), mais il est possible qu'intervienne également IL-6 ; la synthèse s'effectue dans les hépatocytes. On ne sait pas pourquoi seulement certains malades qui ont une production augmentée de SAA, développent une amylose. Jusqu'à présent, on n'a pas trouvé de forme particulière de SAA qui soit plus «amyloïdogène » que d'autres (chez la souris, une seule forme de SAA est incorporée dans la substance amyloïde).

Skinner et al. [3] ont isolé et étudié la protéine AA (8 000 daltons) qui composait l'amylose compliquant une mucoviscidose. La séquence de la protéine est très proche de celle observée pour d'autres protéines AA, avec seulement deux substitutions par de l'acide aspartique à la place de l'asparagine en positions 23 et 75 . Une telle transition, asparagine $\rightarrow$ acide aspartique, est probablement secondaire à une désamination post-traductionnelle. La protéine isolée représente un fragment de $\mathrm{SAA}_{1} \alpha\left(\mathrm{SAA}_{1}\right.$ a deux formes alléliques, $\alpha$ et $\beta$ ); les protéines AA mises en évidence dans la polyarthrite rhumatoïde ont une structure analogue ; en revanche, la protéine AA d'un malade atteint de fiève méditerranéenne familiale semble dériver de $\operatorname{SSA}_{1} \beta$.

J.-P.G.

1. Benson MD. Reactive (secondary) amyloid and its protein precursor. J Lab Clin Med 1988; 112: 405-6.

2. Kluve-Beckerman B, Dwulet FE, Benson MD. Human serum amyloid A, three hepatic m RNAs and the corresponding proteins in one person. J Clin Invest 1988; $82: 1670-5$.

3. Skinner M, Pinnette A, Travis WD, Shwachman $\mathrm{H}$, Cohen AS. Isolation and sequence anlysis of amyloid protein AA from a patient with cystic fibrosis J Lab Clin Med 1988; 112 : 413-7. 